\title{
CLASSIFICAÇÃO E PADRONIZAÇÃO DOS TOMATES CV. CARMEM E DÉBORA DENTRO DA CEAGESP - SP ${ }^{1}$
}

\section{CAROLINE ANDREUCCETTI ${ }^{2}$, MARCOS D. FERREIRA ${ }^{3}$, ANITA S. D. GUTIERREZ ${ }^{4}$, MARCELO TAVARES ${ }^{5}$}

\begin{abstract}
RESUMO: A criação do Programa Brasileiro para a Modernização da Horticultura pela Secretaria de Agricultura e Abastecimento do Estado de São Paulo, em conjunto com a Companhia de Entrepostos e Armazéns Gerais de São Paulo (CEAGESP), determinou a padronização das hortaliças e frutas comercializadas nos seguintes aspectos: grau de coloração, formato, calibres, defeitos e embalagens, um trabalho iniciado em setembro de 1997. O objetivo deste trabalho foi relacionar a classificação fornecida pelo Programa Brasileiro com a realizada pelos atacadistas no mercado da CEAGESP, verificando se as normas estabelecidas estão sendo cumpridas para os tomates cultivares Carmem e Débora (SAKATA SEED). Os resultados mostraram que, para o tomate cultivar Carmem, a partir da análise de teste de hipótese de proporção, as médias dos valores observados não se afastam muito das normas criadas pelo Programa para os tamanhos pequeno e médio. Porém, para o caso do tomate cultivar Débora, encontraram-se, a partir dos resultados, diferenças entre as classificações adotadas, percebendo que os tomates estavam desvalorizados, pois estavam sendo comercializados em padronização inferior às normas indicadas pelo Programa Brasileiro.
\end{abstract}

PALAVRAS-CHAVE: Lycopersicum esculentum Mill., normas de padronização, atacadista.

\section{CLASSIFICATION AND STANDARDIZATION OF TOMATOES CV. CARMEM AND DEBORA IN THE CEAGESP - SÃO PAULO - BRAZIL}

SUMMARY: The creation of the Brazilian Program for the Modernization of the Horticulture by the Secretariat of Agriculture and Supplying of the State of São Paulo at CEAGESP, determined the standardization of fruit and vegetables in the follow aspects: degree of coloration, format, calibers, defects and packing. Therefore, the main goal of this research is to correlate the classification given by the Brazilian Program with the one used by the wholesalers at CEAGESP, verifying if the established norms are being fulfilled for cultivar Carmen and Debora (SAKATA SEED). The results showed, that for cultivar Carmem, for the averages of the observed values it does not move away from the norms created by the Program for sizes small and medium. However, for the case of cultivar Debora, the results showed differences between the adopted classifications. The tomatoes were devaluated, because had been commercialized below of the standardization indicated for the Brazilian Program.

KEYWORDS: Lycopersicum esculentum Mill., standards classification, wholesalers.

\footnotetext{
${ }^{1}$ Extraído do projeto de pesquisa UNIMAC (Unidade Mecânica de Auxílio à Colheita) financiado pela FAPESP.

${ }^{2}$ Eng $^{\mathrm{a}}$ Agrícola, aluna de Mestrado, Faculdade de Engenharia Agrícola, UNICAMP, Caixa Postal 6011, Campinas - SP, e-mail: cline@agr.unicamp.br

${ }^{3}$ Eng ${ }^{-}$Agrônomo, Prof. Doutor, Faculdade de Engenharia Agrícola, UNICAMP, Caixa Postal 6011, Campinas - SP.

${ }^{4}$ Eng ${ }^{\mathbf{a}}$ Agrônoma, CEASGESP-CQH (Centro de Qualidade em Horticultura), Av. Gastão Vidigal, 1946, São Paulo - SP.

${ }^{5}$ Eng $^{\mathrm{O}}$ Agrônomo, UFU, Faculdade de Matemática, Av. João Naves de Ávila, 2121, Uberlândia - MG.

Recebido pelo Conselho Editorial em: 29-10-2003

Aprovado pelo Conselho Editorial em: 15-6-2004
} 


\section{INTRODUÇÃO}

O mercado de hortaliças, em especial a cultura do tomate, tem passado por alterações nos últimos anos e uma delas se refere às cultivares utilizadas. Há uma década, os produtores passaram da utilização de cultivares do grupo Santa Cruz, cultivar Ângela, para a cultivar Santa Clara e, posteriormente, para o cultivo do híbrido Carmem, tipo longa vida (FILGUEIRA, 2000). Atualmente, existem no mercado diversas cultivares disponíveis para cultivo, provenientes de diferentes grupos: salada, italiano, entre outros. Apesar da diversidade de cultivares, os tomates são vendidos sem distinção nas gôndolas de supermercados. Esse comportamento é encontrado para a maioria das frutas e hortaliças que são comercializadas sem padrões de qualidade e sem estarem acondicionadas em embalagens apropriadas. Esse contexto acarreta desvalorização dos produtos e muitas vezes propiciam o aumento dos índices de perdas dentro da cadeia de comercialização. KADER (2002) relata que a classificação e a normatização identificam os padrões de qualidade de um produto, os quais são valiosos instrumentos na comercialização de frutas e hortaliças, proporcionando uma linguagem comum entre produtores, intermediários e consumidores, incentivando pagamentos diferenciados para produtos de melhor qualidade. FAGUNDES \& YAMANISHI (2001) descrevem que, para a comercialização de banana em diferentes estados, o preço do produto está diretamente relacionado à qualidade oferecida.

Desde 1997, as normas de classificação e os padrões de qualidade de frutas e hortaliças frescas estão sendo desenvolvidos com o objetivo de minimizar os principais problemas das cadeias de produção, tais como a inexistência de padrões mensuráveis de qualidade e a melhoria das embalagens (CEAGESP, 2003). Esse trabalho é conhecido por Programa Brasileiro para a Modernização da Horticultura (PB). O Programa Brasileiro, resultado da parceria das Câmaras Setoriais de Frutas e Hortaliças da Secretaria de Agricultura e Abastecimento do Estado de São Paulo e a Companhia de Entrepostos e Armazéns Gerais de São Paulo (CEAGESP), que desenvolve os padrões de comercialização, é avaliado como sendo o único caminho para garantir a competitividade do produto agrícola brasileiro, com menores índices de perdas, preço justo, melhor qualidade e rentabilidade, com possibilidades de agregar valor aos produtos e ainda garantir continuidade de ganho ao produtor, levando a fidelização do consumidor (COMERCIALIZAÇÃO, 1999). O tomate foi o primeiro produto trabalhado por esse Programa e constitui uma das hortaliças de maior importância econômica para o País.

Segundo o Instituto Brasileiro de Geografia e Estatística - IBGE (2003), a produção de tomate no Brasil atingiu 3.595.730 toneladas em 2002, englobando tomate de mesa e para a indústria, e ultrapassando os valores dos anos anteriores, sendo o Estado de São Paulo o maior produtor do Brasil, com cerca de 34,6\% da produção total. AGRIANUAL 2002 (2001) estimou a produção paulista em 638.985 toneladas, sendo a produção da região Sudeste de 1.556.314 toneladas. RESENDE (1995) afirma que aproximadamente dois terços da produção nacional é destinada ao consumo in natura. O preço médio da caixa de tomate, nos últimos cinco anos, foi de US\$4,23, segundo a CEAGESP e divulgado pelo AGRIANUAL 2002 (2001).

Para garantir o abastecimento de tomate de mesa durante todo o ano, os atacadistas da CEAGESP recebem o produto de várias regiões do País em diferentes épocas do ano. Essa diversidade de origem do produto dificulta a padronização. No entanto, as atuais exigências de mercado, tais como melhor qualidade, incluindo aparência, textura, sabor e aroma, valor nutritivo e segurança alimentar, fazem com que os produtores sintam a necessidade das normas em um mercado cada vez mais competitivo.

Para BELÉM (2003), a classificação garante um padrão único para os produtores, atacadistas e consumidores finais, promove maior facilidade na comercialização, dando a possibilidade de que os 
melhores agricultores possam ter seus produtos valorizados, diminuem as perdas, além de garantir ao consumidor elevado padrão de qualidade de frutas e hortaliças.

Portanto, este trabalho teve o objetivo de estabelecer uma relação entre a classificação recomendada pelo Programa Brasileiro com a utilizada pelos atacadistas no mercado da CEAGESP, verificando se as normas preconizadas estão sendo cumpridas para os tomates cultivar Carmem e Débora.

\section{MATERIAL E MÉTODOS}

As avaliações quanto à classificação dos tomates cultivar Débora e Carmem foram realizadas em atacadistas do ETSP - Entreposto Terminal de São Paulo da Companhia de Entrepostos e Armazéns Gerais de São Paulo (CEAGESP), durante o período de setembro a novembro de 2002. Encontram-se no ETSP/CEAGESP, 114 permissionários que comercializam tomate. Procurou-se obter as informações daqueles permissionários com maior representatividade e, para tanto, foi fornecido pela CEAGESP, o Movimento dos Permissionários durante o mês de setembro de 2002, o qual corresponde a uma listagem relacionada ao volume comercializado de tomate de mesa naquele mês. Selecionaramse os atacadistas com maior volume comercializado nesse período, equivalente a $70 \%$ do volume total, perfazendo 24 permissionários. Nesses atacadistas, foram tomados aleatoriamente, 10 frutos por caixa $\mathrm{K}$ (22 kg), com dimensões de $495 \mathrm{~mm}$ de comprimento, $230 \mathrm{~mm}$ de largura e $355 \mathrm{~mm}$ de altura (FILGUEIRA, 1981), perfazendo um total de 360 frutos analisados por cultivar. A classificação dos tomates pertencentes à cultivar Carmem e Débora foi realizada medindo-se os diâmetros transversais dos frutos por meio da utilização de um paquímetro. Compararam-se os diâmetros obtidos no levantamento (classificação mercado) com os estabelecidos pelo Programa Brasileiro (classificação CEAGESP).

Para o tomate cultivar Carmem (formato redondo), a classificação proposta pelo Programa Brasileiro determina como pequenos os frutos com diâmetro entre 50 e $65 \mathrm{~mm}$, médio entre 65 e $80 \mathrm{~mm}$ e acima de $80 \mathrm{~mm}$ considera-se grande. O Programa Brasileiro recomenda que, para tomates com formato oblongo, caso da cultivar Débora, calibres entre 40 e $50 \mathrm{~mm}$ são pequenos, entre 50 e $60 \mathrm{~mm}$ são médios e acima de $60 \mathrm{~mm}$ grandes. A classificação adotada no mercado valoriza o tamanho, pois quanto mais graúdo, maior o valor comercial. Os tamanhos, nesse caso, são: 1A (miúdo), 2A (tamanho intermediário) e 3A (graúdo).

Foi realizado um teste de hipótese para proporção com as hipóteses: $\mathrm{H}_{0}$ quando $\mathrm{p}=1$ e $\mathrm{H}_{1}$ quando $\mathrm{p} \neq 1$, considerando $\mathrm{p}$, por exemplo, a proporção do tomate pequeno na classificação $1 \mathrm{~A}$ na população como sendo $100 \%$ ou proporção igual a 1,0. Se todos os valores forem significativos, aceita-se a hipótese $\mathrm{H}_{1}$ e, portanto, a classificação encontrada não estará seguindo a estabelecida pelo Programa Brasileiro, conforme situação para os diferentes diâmetros.

\section{RESULTADOS E DISCUSSÃO}

A classificação adotada pelos permissionários qualifica os tomates por meio do diâmetro transversal, ou seja, quanto mais graúdo for o fruto, maior valor comercial terá. As denominações de tamanhos diferem entre os atacadistas e o Programa Brasileiro, sendo classificados pelos atacadistas como 1A, 2A e 3A, os quais equivalem no Programa Brasileiro aos tamanhos pequeno, médio e grande, respectivamente. Apesar de a classificação ser uma operação praticada por todos os atacadistas, percebe-se, em uma mesma caixa, grande mistura de padrões de coloração e tamanho.

Dentre os 24 permissionários entrevistados, destaca-se que $58,4 \%$ realizam a classificação manual, 8,3\% classificação por máquinas e 33,3\% utilizam os dois métodos. A classificação realizada, seja manual, seja por máquina, é feita seguindo os parâmetros de coloração e tamanho. Todavia, 
alguns atacadistas $(54,2 \%)$ fazem a reclassificação manual dentro do mercado, pois identificam grande deficiência na padronização de coloração e tamanho dos frutos.

\section{Tomate cultivar Carmem}

Na Tabela 1, são apresentados os resultados obtidos por meio do método estatístico: teste de hipótese de proporção. O número de sucessos corresponde aos diâmetros obtidos no mercado CEAGESP que se enquadravam na classificação do Programa Brasileiro. O parâmetro "p1" equivale a essa proporção e " $n 1$ " o número de amostras. Observa-se que, para as três variáveis (1A, $2 \mathrm{~A}, 3 \mathrm{~A})$, a proporção p1 resultou em valor abaixo de um, logo, descarta-se a primeira hipótese $\left(\mathrm{H}_{0}\right)$, conforme especificado em Material e Métodos.

TABELA 1. Hipótese de proporção para as classificações dadas pelos atacadistas e pelo Programa Brasileiro (CEAGESP) para tomate cv. Carmem.

\begin{tabular}{cccccc}
\hline Variável & $\mathrm{N}^{\circ}$ Sucessos & $\mathrm{p} 1$ & $\mathrm{n} 1$ & zcalc & Sigf $(\mathrm{z})$ \\
\hline Carmem 1A & 90 & 0,818182 & 110 & $-4,94413$ & 0,00000076 \\
Carmem 2A & 156 & 0,866667 & 180 & $-5,26235$ & 0,00000014 \\
Carmem 3A & 25 & 0,357143 & 70 & $-11,225$ & 0 \\
\hline
\end{tabular}

As Figuras 1; 2 e 3 correspondem às comparações entre as classificações. Cada ponto do gráfico corresponde a uma média de diâmetros por caixa medida. Na Figura 1, constata-se que a classificação do mercado (com apenas um ponto fora do padrão) segue aproximadamente as normas estabelecidas pelo Programa Brasileiro, com proporção p1 =0,818 (Tabela 1), ou seja, de uma amostra de 110 tomates medidos, 90 estavam condizentes com a norma.

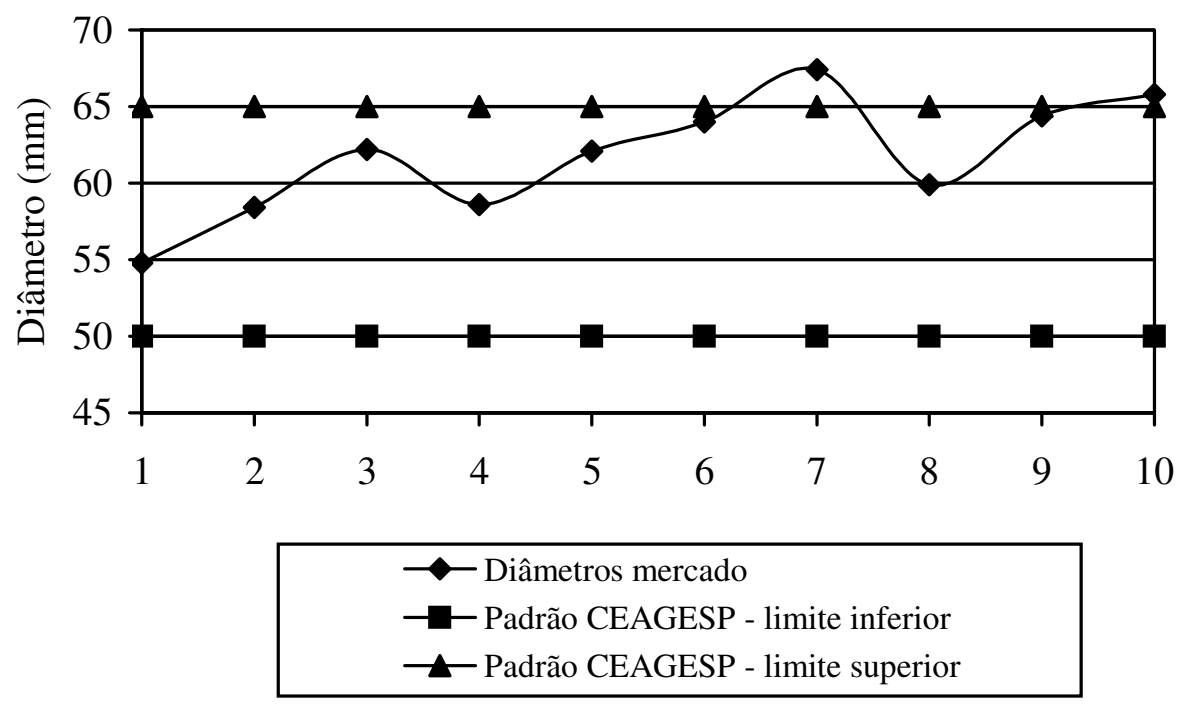

FIGURA 1. Comparação entre a classificação do mercado (1A) e da CEAGESP (pequeno) para tomate cultivar "Carmem".

Na Figura 2, com apenas um ponto fora do padrão, percebe-se que há a tendência da classificação do mercado em seguir as normas do Programa Brasileiro, com proporção p1 $=0,867$ (Tabela 1). Dentro de um conjunto de 180 medições, observa-se que 156 tomates condiziam com a classificação indicada pelo Programa Brasileiro. 


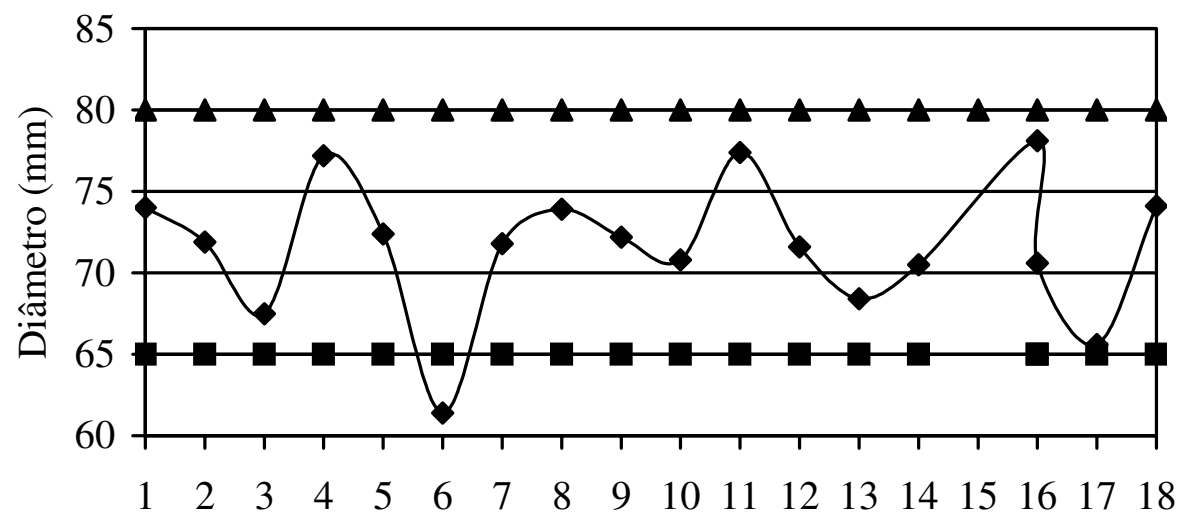

$\neg$ Diâmetros mercado

Padrão CEAGESP - limite inferior

—-Padrão CEAGESP -limite superior

FIGURA 2. Comparação entre a classificação do mercado (2A) e da CEAGESP (médio) para tomate cultivar Carmem.

$\mathrm{Na}$ Figura 3, no entanto, observa-se que a classificação para tomate 3A (grande) está supervalorizada, pois os pontos encontram-se abaixo da linha de limite inferior de padrão, ou seja, os tomates estão sendo vendidos como 3A, mas, na verdade, seguindo a classificação do Programa Brasileiro, esses tomates deveriam estar sendo comercializados como 2A (tamanho médio, com diâmetro entre 65 e $80 \mathrm{~cm}$ ).

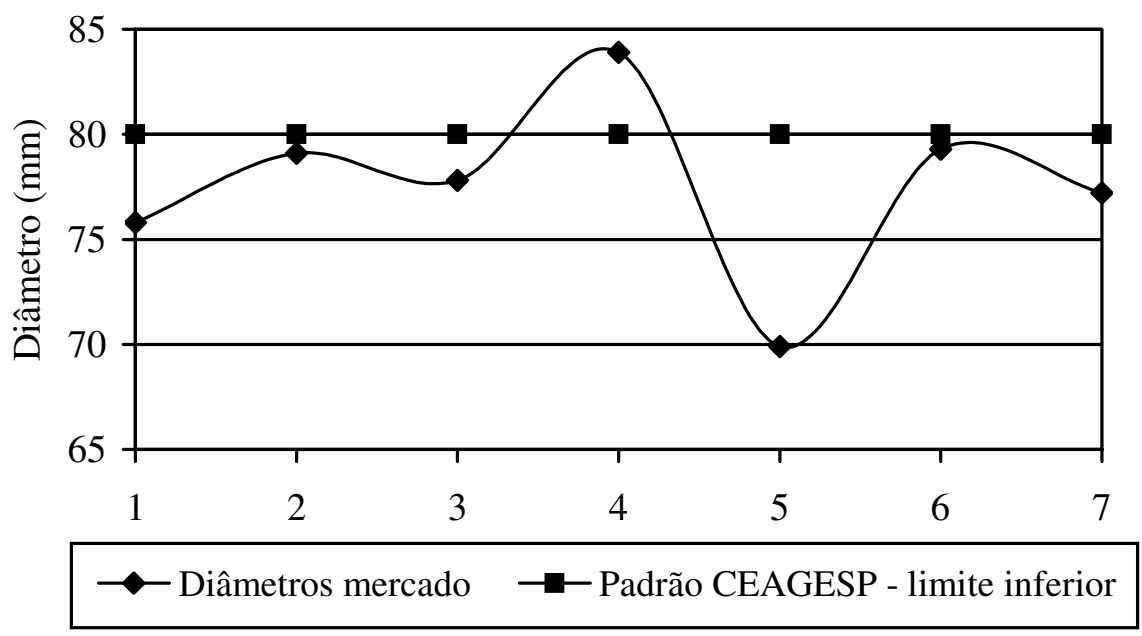

FIGURA 3. Comparação entre a classificação do mercado (3A) e da CEAGESP (grande) para tomate cultivar Carmem.

\section{Tomate cultivar Débora}

Para a análise da padronização dos diâmetros referentes ao tomate cultivar Débora, utilizaram-se os diâmetros obtidos por meio dos tomates da CEAGESP. A partir da análise de hipótese para proporção, obteve-se a Tabela 2, o número de sucessos corresponde aos diâmetros obtidos no mercado CEAGESP que se enquadravam na classificação do Programa Brasileiro. O parâmetro "p1" equivale a 
essa proporção e "n1" o número de amostras. Observa-se que para as três variáveis (1A, 2A, 3A), a proporção p1 resultou em valor abaixo de um, logo descarta-se a primeira hipótese $\left(\mathrm{H}_{0}\right)$.

TABELA 2. Comparação entre as classificações dadas pelos atacadistas e Programa Brasileiro (CEAGESP) para tomate cv. Débora.

\begin{tabular}{cclccc}
\hline Variável & $\mathrm{N}^{\circ}$ Sucessos & \multicolumn{1}{c}{$\mathrm{p} 1$} & $\mathrm{n} 1$ & zcalc & Sigf $(\mathrm{z})$ \\
\hline${\text { Débora } 1^{\mathrm{a}}}_{\text {Débora }^{\mathrm{a}}}^{\mathrm{a}}$ & 27 & 0,245455 & 110 & 18,3888 & 0 \\
Débora $^{\mathrm{a}}$ & 85 & 0,5 & 170 & $-13,0384$ & 0 \\
\hline
\end{tabular}

A partir das Figuras 4; 5 e 6, podem-se observar os valores dos diâmetros dos tomates do padrão CEAGESP e os resultados medidos no mercado. Cada ponto do gráfico corresponde à média de diâmetros por caixa medida. Como as significâncias (sigf z) foram menores que 0,05 , há discrepância entre as classificações. Na Figura 4, demonstra-se que há, dentre os diâmetros medidos, três pontos dentro do padrão indicado pelo Programa Brasileiro, ou seja, os pontos acima do limite superior correspondem ao tamanho médio (diâmetro entre 50 e $60 \mathrm{~cm}$ ), logo os tomates (aqueles fora do padrão) que estavam sendo vendidos como 1A pelos atacadistas, poderiam estar sendo comercializados como $2 \mathrm{~A}$, resultando, portanto, em um produto desvalorizado (conforme Tabela 2, de uma amostra de 110 tomates, apenas 27 estavam classificados adequadamente). SARGENT et al. (1991) relatam que tomates de menor tamanho estão mais sujeitos a classificação incorreta do que frutos de maior tamanho.

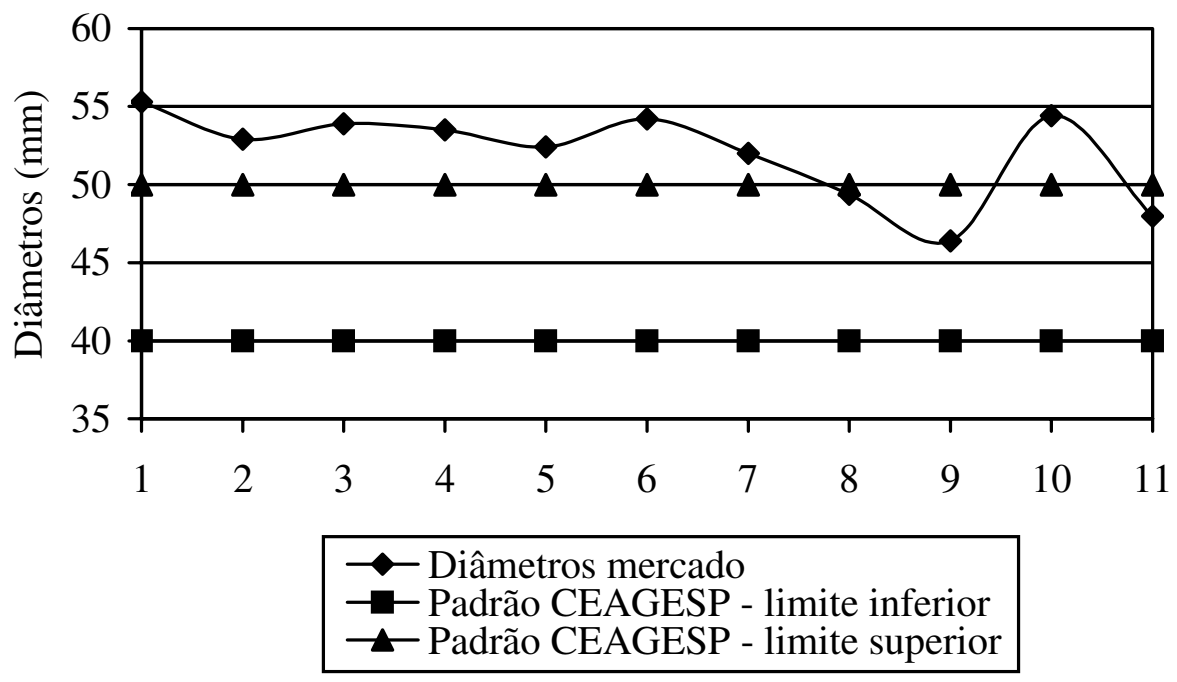

FIGURA 4. Comparação entre a classificação mercado (1A) e da CEAGESP (pequeno) para tomate cultivar Débora.

Na Figura 5, observa-se grande variação dos diâmetros dos tomates obtidos. Na Tabela 2 (Débora 2A), verifica-se que a proporção $\mathrm{p} 1=0,5$ indica que metade dos tomates obtidos se encontravam dentro da norma. Aqueles frutos fora dos limites estabelecidos pelo Programa Brasileiro estavam desvalorizados, pois poderiam estar sendo vendidos como tomate tamanho grande (3A), com diâmetro superior a $60 \mathrm{~cm}$. 


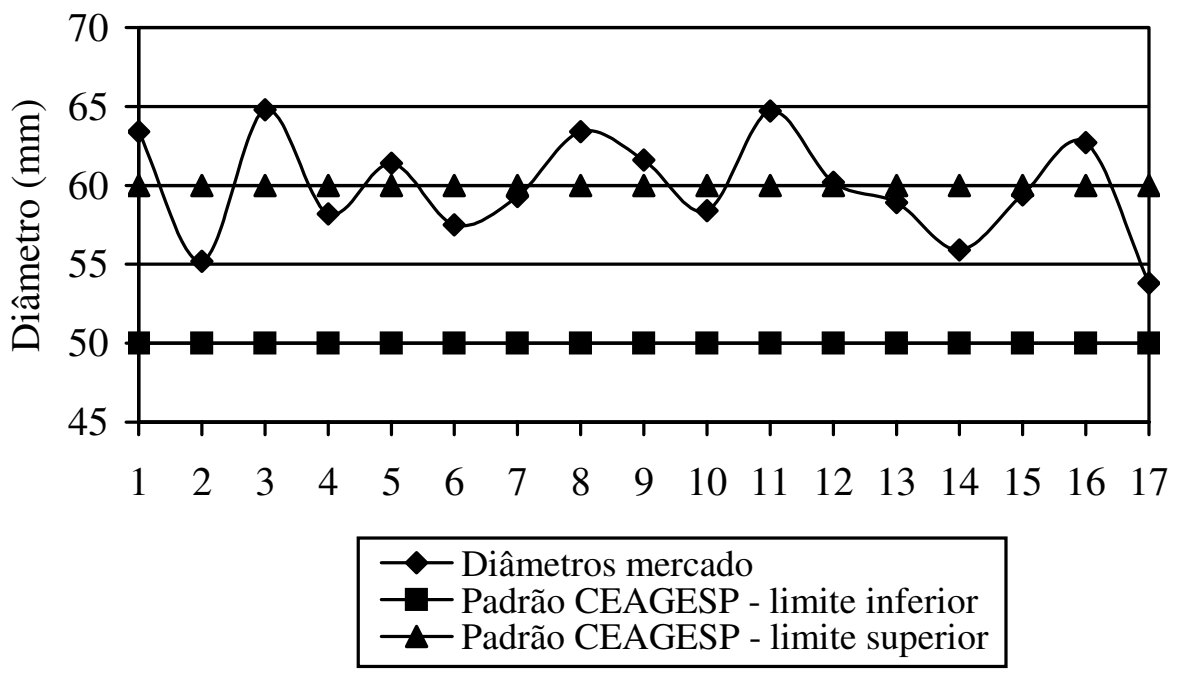

FIGURA 5. Comparação entre a classificação do mercado (2A) e da CEAGESP (médio) para tomate cultivar Débora.

A análise da Figura 6 demonstra que, para a cultivar Débora 3A, os tomates estavam mais adequados ao padrão da CEAGESP do que em relação às outras classificações determinadas, alcançando uma proporção de 0,8375; ou seja, conforme Tabela 2, numa amostra de 80 frutos, 67 deles encontravam-se dentro das normas exigidas pelo Programa Brasileiro.

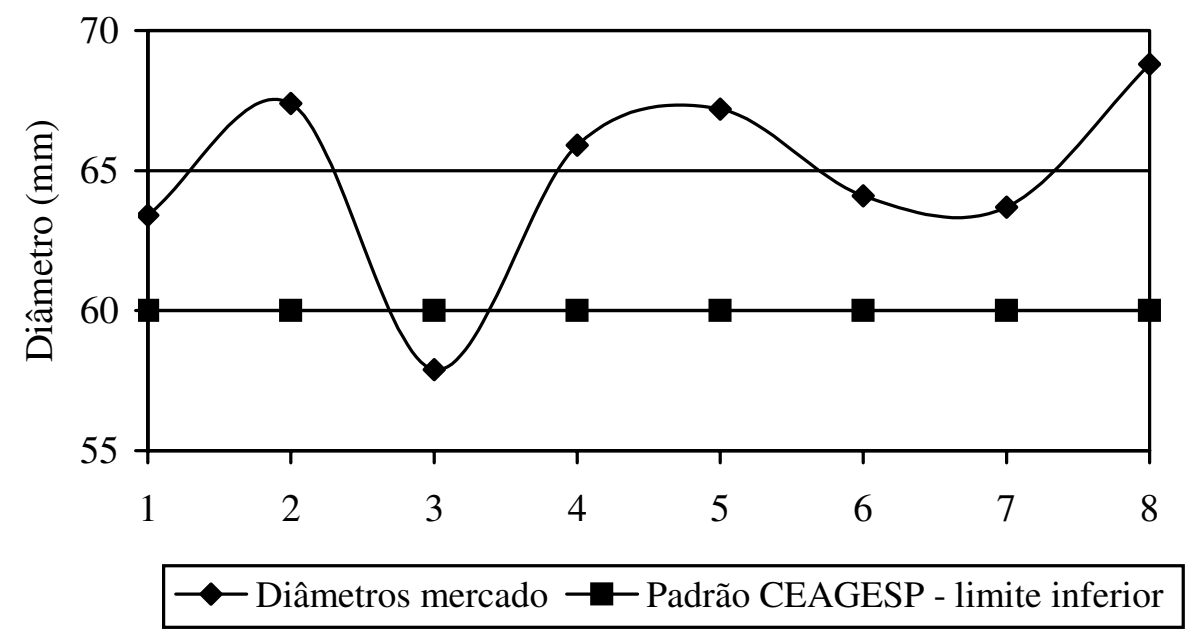

FIGURA 6. Comparação entre a classificação do mercado (3A) e da CEAGESP (grande) para tomate cultivar Débora.

Pode-se, a partir dos resultados da Tabela 2 e ainda das Figuras 4; 5 e 6, observar que existem proporções de frutos pequenos, médios e grandes, embora em quantidades diferentes. Porém, o mercado procura atender à diversidade de preferência por diferentes tamanhos de tomates, sendo comercializados os três tipos mesmo que ainda fora do padrão. Percebe-se, diante dos resultados apresentados, a grande diversificação que existe frente aos parâmetros de classificação. LOPEZ CAMELO et al. (2003), em trabalho realizado com cebola, também encontraram dificuldades de estabelecer padrões de classificação para esse produto. Afirmaram que as diferenças de classificação 
podiam ser atribuídas principalmente à seqüência de operações e à velocidade das correias de transporte. Esse argumento utilizado pode ser refletido no caso da classificação de tomate, uma vez que também é feita a utilização de máquinas para proceder a classificação do produto. Outro fator que interfere nesse processo, é a classificação manual, a qual está sujeita à subjetividade de cada trabalhador.

Comparando-se os resultados obtidos para as cultivares Carmem e Débora, observa-se, para a segunda, maior diferença entre a classificação utilizada pela CEAGESP e a utilizada pelo mercado. SARGENT et al. (1991) relatam que a eficiência para classificação em tomates com formato oblongo é mais difícil de obter, devido à dificuldade da mensuração do diâmetro médio do fruto ocorrer em algumas ocasiões na transversal e, em outras, na longitudinal. Constata-se que, para a cultivar Carmem, as normas, de maneira geral, foram atendidas conforme especificação do Programa Brasileiro, exceto para os tomates tamanho $3 \mathrm{~A}$, os quais estavam supervalorizados. A grande variabilidade encontrada em termos de padronização, principalmente para o tomate cultivar Débora, pode ser explicada pela diferenciação do tipo de classificação que é realizada, isto é, alguns atacadistas recebem produtos classificados manualmente, por máquinas ou, ainda, por ambos os sistemas. Esse fato acarreta desuniformidade de um lote, principalmente quando a classificação é feita manualmente, a qual depende da subjetividade de cada trabalhador. Acredita-se, ainda, que sendo a cultivar Carmem de formato redondo, seja mais fácil sua classificação, permitindo melhor visualização quanto ao seu diâmetro, diferentemente do que acontece com a cultivar Débora, para a qual provavelmente se estabeleça melhor visualização quanto a sua maior dimensão, que é representada pela altura, e não pelo seu diâmetro.

\section{CONCLUSÃO}

Para tomates de formato redondo, existe uma maior correlação entre a classificação utilizada pelos atacadistas e a do Programa Brasileiro, do que aquela realizada em tomates de formato oblongo.

\section{AGRADECIMENTOS}

À FAPESP, pelo apoio financeiro na realização deste trabalho, e ao Centro de Qualidade em Horticultura - CQH, CEAGESP.

\section{REFERÊNCIAS}

AGRIANUAL 2002: anuário da agricultura brasileira. São Paulo: FNP Consultoria e Comércio, 2001. $536 \mathrm{p}$.

BELÉM, M.Q. de. Padronização das frutas. Revista Agroamazônica. Disponível em: <http://www.revistaagroamazonia.com.br/12fruticultura-04.htm>. Acesso em: 10 set. 2003.

COMPANHIA DE ENTREPOSTOS E ARMAZÉNS GERAIS DE SÃO PAULO. Centro de Qualidade em Horticultura. Programa Brasileiro para Modernização da Horticultura: Normas de classificação de tomates. São Paulo, 2003. 3 p. (CQH Doc. 26)

COMERCIALIZAÇÃO ponto fraco da olericultura brasileira. Unesp Rural, Jaboticabal, v.15, n.3, p.59, 1999.

FAGUNDES, G.R.; YAMANISHI, O.K. Quantidade e preços da banana-prata comercializada nas CEASAS do Distrito Federal, São Paulo, Belo Horizonte e Rio de Janeiro, no período de 1995 a 1999. Revista Brasileira de Fruticultura, Jaboticabal, v.23, n.3, p.593-6, dez. 2001.

FILGUEIRA, F.A.R. Manual de olericultura: cultura e comercialização de hortaliças. 2. ed. São Paulo: Editora Ceres. 1981. v.1, 338 p. 
FILGUEIRA, F.A.R. Novo Manual de Olericultura: agrotecnologia moderna na produção e comercialização de hortaliças. Viçosa: Editora da Universidade Federal de Viçosa, 2000. 402 p.

INSTITUTO BRASILEIRO DE GEOGRAFIA E ESTATÍSTICA. Produção Agrícola 2000 e 2001. Disponível em: <http://www.ibge.gov.br>. Acesso em: 17 mar. 2003.

KADER, A.A. Standardization and inspection of fresh fruits and vegetables. In: KADER, A.A. Postharvest technology of horticultural crops. 3. ed. California: University of California, Agricultural and Natural Resources, 2002. p.287-9. (Publication, 3311)

LOPEZ CAMELO, A.F.; HORVITZ, S.; GOMEZ, P.A. An approach for the evaluation of efficiency of onion packinghouse operations. Horticultura Brasileira, Brasília, v.21, n.1, p.51-4, 2003.

RESENDE, J.M. Qualidade pós-colheita de dez genótipos de tomate do grupo multilocular. 1995. 90 f. Dissertação (Mestrado em Ciência dos Alimentos) - Faculdade de Ciência dos Alimentos, Universidade Federal de Lavras, Lavras, 1995.

SARGENT, S.A.; BRECHT, J.K.; TALBOT, M.T.; ZOELLNER, J.J. Performance of perforated-belt sizers as affected by size standards for fresh market tomatoes. Applied Engineering in Agriculture, St. Joseph, v.7, n.6, p.725-8, 1991. 